\title{
Solubilization of Water by Dialkyl Sodium Sulfosuccinates in Hydrocarbon Solutions
}

\author{
SYLVAN G. FRANK ${ }^{1}$ AND GEORGE ZOGRAFI \\ College of Pharmacy, The University of Michigan, Ann Arbor, Michigan 48103
}

Received June 10, 1968

\begin{abstract}
The apparent solubilization of water in hydrocarbon solvents by the addition of $0.1 \%$ to $2.0 \% \mathrm{w} / \mathrm{w}$ di-(2-ethylhexyl) sodium sulfosuecinate (AOT) bas been studied. In $n$-octane and $n$-decane, transitions of elear-turbid-clear-turbid were observed with increasing water content, whereas in dodecane, tetradecane, hexadecane, cyclohexane, and toluene only one clear-turbid transition was seen. Separation of the first turbid region in $n$-octane and $n$-decane gave two clear phases. Direct analysis indicates a hydrocarbon phase containing surfactant-solubilized water and an aqueous phase containing some surfactant. Increasing amounts of water partition between these phases until a second clear region appears. Separation of phases in the second turbid region in $n$-octane and $n$-decane and in the turbid region of the other solvents produces a pure hydrocarbon phase and a phase containing a complex aggregate of $\mathrm{AOT} /$ water/hydrocarbon. Solubilization of water in $n$-octane solutions of di-(n-octyl) sodium sulfosuccinate and di-(n-hexyl) sodium sulfosuccinate is greatly reduced when compared to AOT. This suggests a significant effect due to the ethyl side chain in the AOT molecule.
\end{abstract}

\section{INTRODUCTION}

The micellar behavior and water-solubilizing ability of di-(2-ethylhexyl) sodium sulfosuccinate (Aerosol OT) in a variety of nonaqueous solvents have been studied extensively (1-8). Of particular interest to the authors was the study of Higuchi and Misra (8), which reported significant amounts of water solubilized in a series of $n$-aliphatic hydrocarbon solvents containing Aerosol OT. The large amounts of water solubilized and a marked dependency on solvent chain length were comparable to observations made by Cooke and Schulman for microemulsion formation in a series of $n$-aliphatic hydrocarbon solvents (9). This, plus the interesting lyotropic liquid crystalline behavior of Aerosol OT when in contact with water $(10,11)$, suggested that these three-

1 National Institutes of Health Training Grant Predoctoral Fellow, IT-GM-1367; present address: Sehool of Pharmacy, Duquesne University, Pittsburgh, Pennsylvania 15219. component systems might be useful models for gaining more fundamental information about the more complex microemulsion systems.

Solubilization of water was measured in $0.1 \%$ to $2.0 \% \mathrm{w} / \mathrm{w}$ Aerosol OT-hydrocarbon solvent solutions, and the influence of temperature and electrolyte considered. Two closely related surfactants, di-( $n$-octyl) sodium sulfosuccinate and di-(n-hexyl) sodium sulfosuccinate, were utilized to observe the effect of chemical structure on water solubilization. These latter two derivatives do not exhibit the significant lyotropic liquid crystalline behavior noted for Aerosol OT at ambient temperatures (10).

\section{EXPERIMENTAL}

Materials. Di-(2-ethylhexyl) sodium sulfosuccinate (Aerosol OT, AOT), di-( $n$-hexyl) sodium sulfosuccinate (Aerosol MA, AMA), and di-(n-octyl) sodium sulfosuccinate (OOT) were synthesized according to a procedure described previously $(12,13)$.

Journal of Colloid and Interface Science, Vol. 29, No. 1, January 1969 
Whereas AOT exists in an amorphous state at room temperature (10), AMA and OOT are crystalline compounds which were recrystallized according to the method of Williams et al. (14). Upon heating AOT gave a broad softening range of about $140^{\circ}$ $160^{\circ} \mathrm{C}$, whereas AMA and OOT had melting points of $237^{\circ}$ and $265^{\circ} \mathrm{C}$, respectively. Surface tension measurements in distilled water at $25^{\circ} \mathrm{C}$ indicated no minima and c.m.c. values of $2.45 \times 10^{-3} M$ for $\mathrm{AOT}, 6.8 \times$ $10^{-4} M$ for OOT, and $1.24 \times 10^{-2} M$ for AMA, all in good agreement with previously reported values $(3,15)$.

The hydrocarbon solvents, $n$-octane, dodecane, and hexadecane (Aldrich Chemical Co.), and $n$-decane and tetradecane (Eastman Organic Chemicals), were treated according to the method of Gillap et al. (16). The cyclohexane and toluene (Matheson, Coleman and Bell) were spectroquality and were twice distilled. Specific gravities determined for all solvents were in excellent agreement with previously reported values (17, 18). The water used in this study was twicedistilled.

Procedure-Water Solubilization. Solutions of the surfactant in hydrocarbon solvent were prepared at concentrations of $0.1 \%$, $0.25 \%, 0.5 \%, \quad 1.0 \%$, and $2.0 \% \mathrm{w} / \mathrm{w} \%$. Water was then introduced to give a wide range of water-to-surfactant molar ratios. Sample tubes were placed in a constanttemperature bath and rotated for 24 hours at about $40 \mathrm{rpm}$. This amount of time was found to be more than sufficient to attain equilibrium. After being removed from the bath the sample tubes were observed individually against a white light for turbidity. Turbidity was defined as any increase in opacity from a colorless-transparent reference, which was a sample of the anhydrous surfactant solution used in preparing the hydrous systems. It was important to observe the samples within 15 seconds after removal to prevent slight temperature changes, as it was found that the appearance of the samples was very sensitive to temperature fluctuation, particularly at the higher temperatures.

The effect of various electrolytes on the water-solubilizing capacity of AOT in $n$ - octane at $25^{\circ} \mathrm{C}$ was determined by repeating the above procedure using aqueous solutions of reagent grade sodium chloride, lithium chloride, and sodium sulfate, all dried in air at $100^{\circ} \mathrm{C}$ for 24 hours prior to use. The following solutions were used: $0.01 M$ and 0.05 $M \mathrm{NaCl}, 0.01 M$ and $0.05 M \mathrm{LiCl}$, and 0.005 $M \mathrm{Na}_{2} \mathrm{SO}_{4}$.

Phase Analysis of Turbid Regions. In the previously described experiments it was observed that increasing the ratio of moles of water per mole of surfactant produced solubilization followed by the appearance of $a$ second phase. For reasons to be described below, analyses for AOT and water in $n$ octane, dodecane, and hexadecane systems were performed to determine the distribution of components in both phases. The samples for analysis were prepared by adding various quantities of water to $40 \mathrm{ml}$ of a $2 \% \mathrm{w} / \mathrm{w}$ AOT solution in 50-ml centrifuge tubes. The amount of water and the concentration of AOT were chosen to provide an amount of separated phase that could be analyzed accurately. The sample tubes were equilibrated for 24 hours at $25^{\circ} \pm 0.1^{\circ} \mathrm{C}$ and then centrifuged at $2400 \mathrm{rpm}$ for 30 minutes. This gave separation of the two phases without an emulsion layer at the interface. Since the volume of the lower layer was considerably less than that of the upper layer, it was necessary to combine the lower layers of four samples in order to obtain a workable amount of lower layer. After volume measurements, most of the upper layer in each sample tube was drawn off, and the remaining contents were combined in a single centrifuge tube. This was centrifuged and the upper layer entirely removed, leaving the combined lower layers. All analyses were run in triplicate, and precision was within $\pm 1 \%$. The volume of the upper layer was measured on the individual samples prepared for analysis. The location of the interface between upper and lower layers was noted on the graduated scale of the centrifuge tube and marked with a fine wax line. The volume of each layer was then determined by adding octane from a buret to each mark, octane being used to reduce curvature. Virtually identical results for the volume of the lower layer were found by measuring 
individual tubes and by doing this with the combined samples.

Specific gravity and AOT content were measured for both layers, but because of limitations in sensitivity water determination by the Karl Fischer method was carried out only on the upper layer; the water content of the lower layer was taken as the difference between that added to the system and that found in the upper layer.

\section{RESULTS AND DISCUSSION}

Solubilization of Water by AOT. As seen in Table I the amount of water solubilized by AOT before turbidity is first produced is influenced significantly by the particular hydrocarbon solvent utilized. These differences are most apparent as the concentration of AOT is increased. Note in particular that in the series of $n$-aliphatic solvents, solubilization passes through a maximum at dodecane, and that by comparison in cyclohexane and toluene it is quite low.

One interesting phenomenon that has not been observed for these systems before is the appearance of several additional phase transitions in $n$-octane and $n$-decane when the amount of water is increased beyond that listed in Table I. In order to observe the factors influencing the various phase transitions, it is convenient to express the data on the basis of moles of water per mole of AOT as a function of AOT concentration. This can be seen in Fig. 1 for the $n$-octane system at $25^{\circ} \mathrm{C}$, which passes through four regions as

\section{TABLE I}

Efect of Solvent and Aerosol OT Conclentration on the Maximum Amount of Water Solubilized in the Colorless Region at $25^{\circ} \mathrm{C}$

\begin{tabular}{lrrrrr}
\hline \multirow{2}{*}{\multicolumn{1}{c}{ Solvent }} & \multicolumn{5}{c}{$\begin{array}{c}\left(\mathrm{ml} \text { water } \times 10^{2}\right)^{a} \\
\text { w/w \% Aerosol } \mathrm{O}^{\mathrm{T}}\end{array}$} \\
\cline { 2 - 6 } & \multicolumn{1}{c}{0.1} & 0.25 & 0.5 & \multicolumn{1}{c}{1.0} & \multicolumn{1}{c}{2.0} \\
\hline$n$-Octane & 0.5 & 1.3 & 2.5 & 5.0 & 10.2 \\
$n$-Decane & 0.5 & 1.3 & 2.8 & 6.3 & 12.7 \\
Dodecane & 0.7 & 1.8 & 4.3 & 10.2 & 22.3 \\
Tetradecane & 0.5 & 1.1 & 2.3 & 4.0 & 8.1 \\
Hexadecane & 0.4 & 0.7 & $\mathbf{1 . 3}$ & 2.6 & $\mathbf{5 . 5}$ \\
Cyclohexane & 0.2 & 0.6 & 1.1 & 3.1 & 6.4 \\
Toluene & 0.2 & 0.4 & 0.7 & 2.3 & 4.1 \\
\hline
\end{tabular}

${ }^{a}$ Per $10 \mathrm{ml}$ of AOT/solvent solution

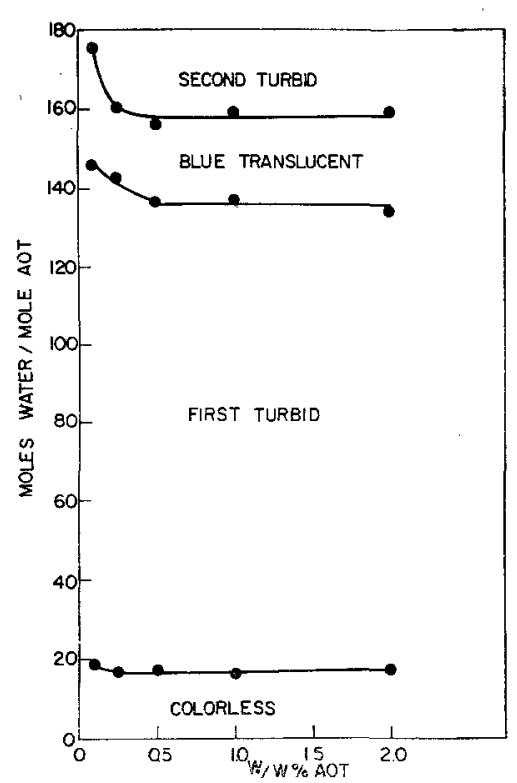

FIG. 1. Phase transitions in a water/Aerosol $\mathrm{oT} / n$-octane system at $25^{\circ} \mathrm{C}$.

the amount of water per amount of AOT is increased. In all such plots the systems have been corrected for the solubility of water in a particular solvent (19). The first region is colorless and exhibits a blue Tyndall beam of increasing intensity as the transition into the first turbid region is approached. The transition is sharp from colorless to gray and as the water content is further increased the system becomes gray-white opaque. Upon centrifugation at $2400 \mathrm{rpm}$ for 30 minutes or standing for several days, two clear layers are formed. Observation of samples in this region, after phase separation, reveals that the upper layer is colorless at the lower ratios of water to AOT but as the water content is increased this layer becomes slightly blue. At the upper limit of the region, the upper layer has a distinct blue translucent color. The colorless lower layer, which occupies a small fraction of the total volume of the system, increases in volume to a maximum about midway through this region, and then decreases until it disappears at the transition into the blue translucent region.

The transition into the one-phase blue translucent region is sharp; an increment of only a fraction of a mole of water per mole of AOT produces a distinct clearing of the sys- 
tem. Samples with increasing water content show an increasing opacity as the second turbid region is approached and then, again with a very small increment of water, marked turbidity occurs. Upon centrifugation at $2400 \mathrm{rpm}$ for 1 hour a colorless upper layer and a blue-gray lower layer are pro-

\section{TABLE II}

Effect of Temperature and Aerosol OT Concentration on the Phase Transitions in a Water/Afrosol OT/ $n$-Octane System

\begin{tabular}{|c|c|c|c|c|c|}
\hline \multirow{2}{*}{$\begin{array}{c}\text { Temper- } \\
\text { ature } \\
\left({ }^{\circ} \mathrm{C}\right)\end{array}$} & \multicolumn{5}{|c|}{$\left(\mathrm{ml} \text { water } \times 10^{2}\right)^{a} \mathrm{w} / \mathrm{w} \%$ Aerosol OT } \\
\hline & 0.1 & 0.25 & 0.5 & 1.0 & 2.0 \\
\hline
\end{tabular}

Transition: Colorless to First Turbid

$\begin{array}{llllll}25 & 0.5 & 1.3 & 2.5 & 5.0 & 10.2 \\ 35 & 0.5 & 1.2 & 2.5 & 5.5 & 11.2 \\ 45 & 0.5 & 1.3 & 2.9 & 6.0 & 14.1 \\ 55 & 0.7 & 1.9 & 4.1 & - & -\end{array}$

Transition: First Turbid to Blue Translucent

$\begin{array}{rrrrrc}25 & 4.1 & 10.1 & 19.1 & 39.1 & 78.9 \\ 35 & 2.9 & 7.1 & 13.4 & 26.2 & 52.7 \\ 45 & 2.1 & 5.0 & 9.5 & 18.2 & 34.8 \\ 55 & 1.3 & 2.9 & 5.1 & - & -\end{array}$

Transition: Blue Translucent to Second Turbid

$\begin{array}{rrrrrr}25 & 4.7 & 11.4 & 22.4 & 45.1 & 90.3 \\ 35 & 4.1 & 9.1 & 17.4 & 34.3 & 68.8 \\ 45 & 3.1 & 7.0 & 13.3 & 26.8 & 52.8 \\ 55 & 2.1 & 4.9 & 10.1 & 20.6 & 41.7\end{array}$

a Per $10 \mathrm{ml}$ AOT- $n$-octane system.

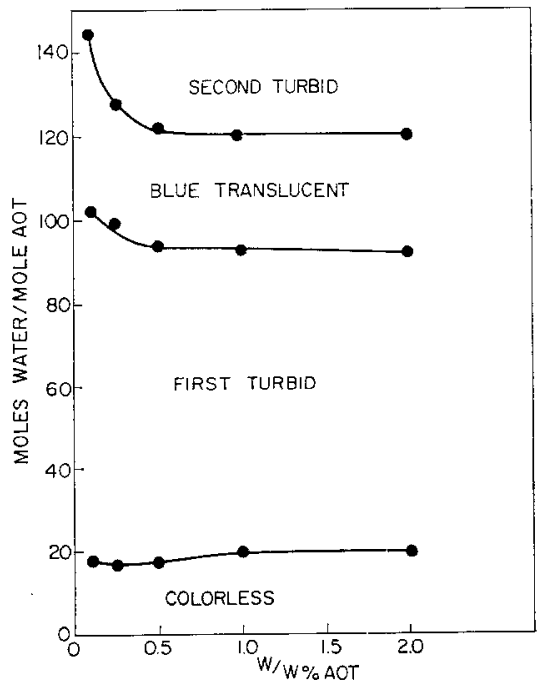

Fig. 2. Phase transitions in a water/Aerosol $\mathrm{OT} / n$-octane system at $35^{\circ} \mathrm{C}$.

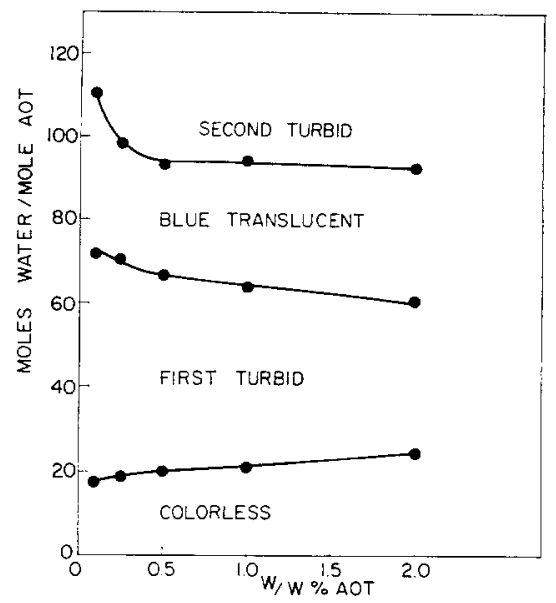

FIG. 3. Phase transitions in a water/Aerosol $\mathrm{OT} / n$-octane system at $45^{\circ} \mathrm{C}$.

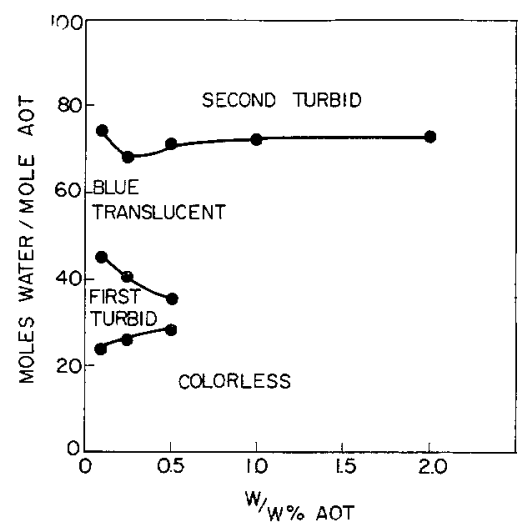

FIG. 4. Phase transitions in a water/Aerosol $\mathrm{O} \mathrm{T} / n$-octane system at $55^{\circ} \mathrm{C}$.

duced. This lower layer has a gelatinous consistency, whereas in the first turbid region the lower layer is more fluid and clear.

Increased temperature produces significant effects on the three phase transitions. As seen in Table II, as the temperature increases, slightly more water can be solubilized before the transition into the first turbid region occurs, whereas less water is required to produce the other transitions at higher temperatures. Figures 2, 3, and 4 show that increased temperature tends to produce a decrease in the extent of the first turbid region with increasing AOT concentration, until at $55^{\circ} \mathrm{C}$ the first turbid region is absent at $1 \%$ and $2 \%$ AOT. The transition defining the limits of this region actually 
appears to merge before $1 \%$ AOT. The transition into the second turbid region remains independent of concentration, as at lower temperatures, although it decreases to a lower molar ratio with each increase in temperature.

As mentioned previously, of the solvents studied, only $n$-octane and $n$-decane show several phase transitions as the ratio of moles of water per mole of AOT is increased. At $25^{\circ} \mathrm{C}$ (Fig. 5) the water $/ \mathrm{AOT} / n$-decane system shows the same four regions as in $n$-octane. However, the molar ratios at which the transitions occur are different; the transition into the first turbid region occurs at higher molar ratios, whereas the other transitions occur at lower molar ratios. At $35^{\circ} \mathrm{C}$ (Fig. 6) the extent of the first turbid region decreases with increasing $\mathrm{AOT}$ concentration until no such region is observed at a concentration of $2 \% \mathrm{w} / \mathrm{w}$ AOT. Only a single transition from colorless to turbid is seen at all AOT concentrations at $45^{\circ} \mathrm{C}$ (Fig. 7). It may be noted in Figs. 5, 6, and 7 that an irregular pattern of behavior occurred at the two boundaries of the blue translucent region for the lower AOT concentrations. This was quite reproducible, although reasons for this observation are not apparent.

The remaining water/AOT/normal aliphatic hydrocarbon solvent systems (Fig.

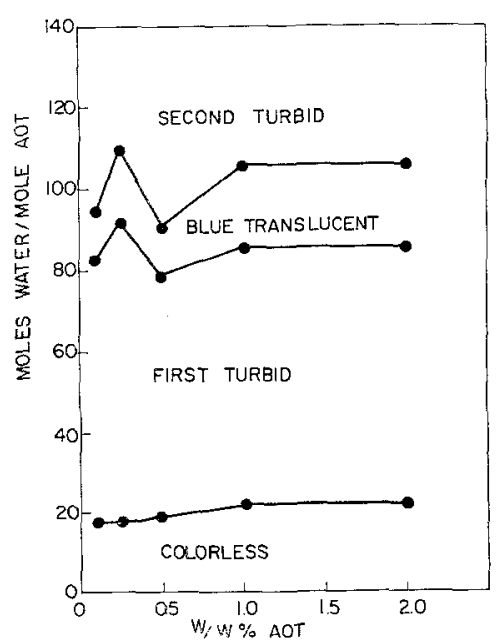

Fig. 5. Phase transitions in a water/Aerosol O $\mathrm{T} / n$-decane system at $25^{\circ} \mathrm{C}$.

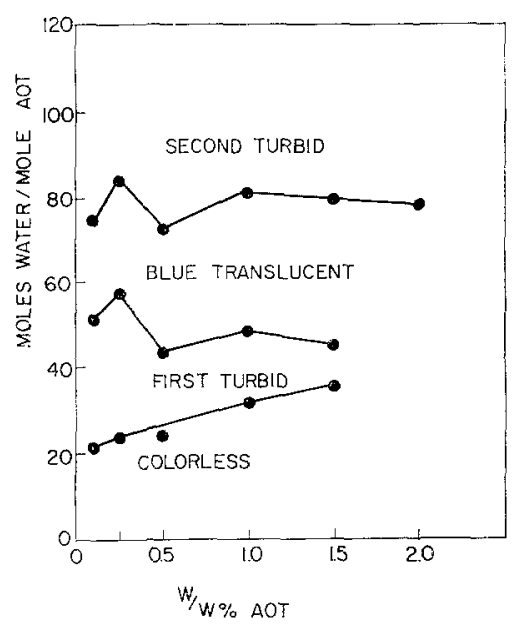

Fig. 6. Phase transitions in a water/Aerosol $\mathrm{O}^{\prime} \mathrm{T} / n$-decane system at $35^{\circ} \mathrm{C}$.

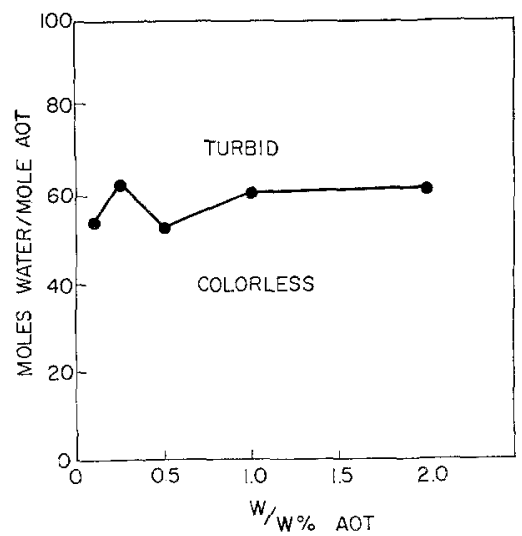

Frg. 7. Phase transitions in a water/Aerosol $\mathrm{OT} / n$-decane system at $45^{\circ} \mathrm{C}$.

8) give a single colorless to turbid transition at all temperatures and AOT concentrations. Except for the dodecane system at $25^{\circ} \mathrm{C}$ there is a slight slight increase in molar ratios at which the transition occurs with increasing temperature. The water/AOT/ cyclohexane (Fig. 9, top) and water/AOT/ toluene (Fig. 9, bottom) systems also give a single colorless to turbid transition. The water-solubilizing capacity of AOT in cyclohexane is similar to that in hexadecane, whereas the toluene systems exhibit the lowest over-all water solubilization of all the solvents used in this study. Notice, however, the rather unusually high efficiency at the low AOT concentrations in toluene. 
Analysis of the Separated Phases. First Turbid Region of Water/AOT/Octane. The results of the analysis of upper and lower layers produced when samples in this region were centrifuged are given in Table III. The
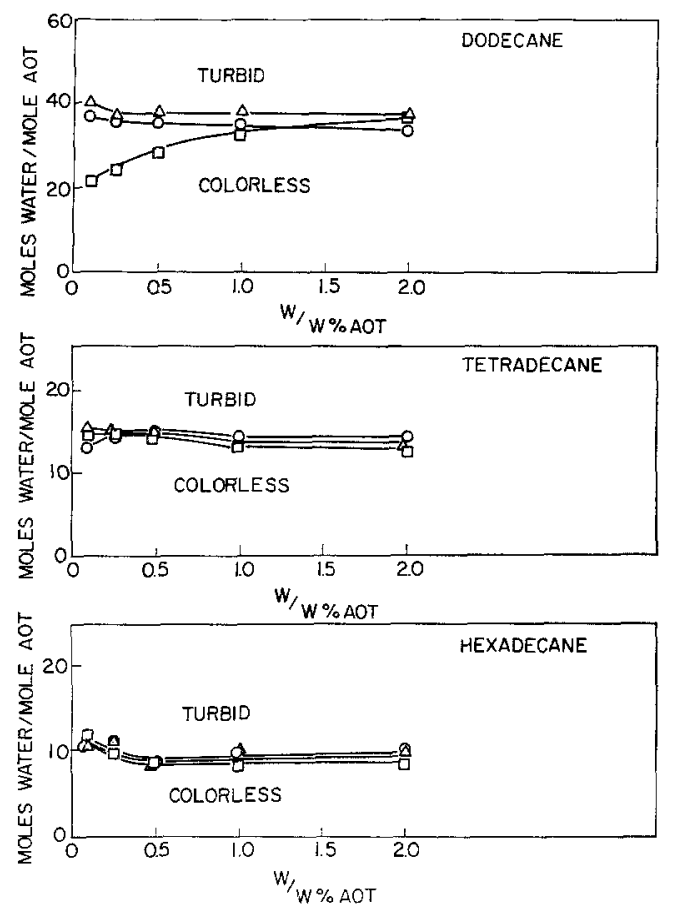

FIG. 8. Phase transition in a water/Aerosol OT system in dodecane (top), tetradecane (mid$d l e$ ), and hexadecane (bottom) at $25^{\circ} \mathrm{C}(\square), 35^{\circ} \mathrm{C}$ $(\triangle)$, and $45^{\circ} \mathrm{C}(\mathrm{O})$.

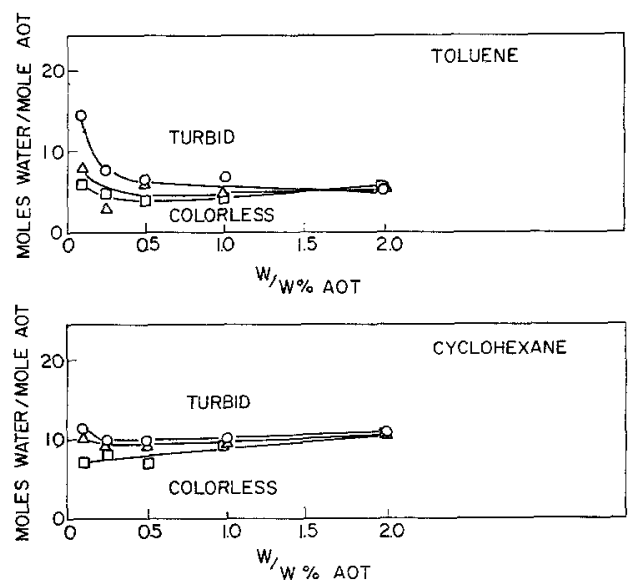

FIG. 9. Phase transition in a water/Aerosol OT system in toluene (top) and cyclohexane (bottom) at $25^{\circ}(\square), 35^{\circ}(\triangle)$, and $45^{\circ}(O)$.
TABLE III

Analysis of the First Turbid Region of Water/Aerosol OT/ $n$-Octane at $25^{\circ} \mathrm{C}$

\begin{tabular}{|c|c|c|c|c|}
\hline$\frac{\text { Moles water }}{\text { mole AOT }}$ & $\underset{(m l)}{\text { Volume }}$ & $\begin{array}{l}\text { Specific } \\
\text { gravity }\end{array}$ & $\begin{array}{c}\text { Water } \\
(\mathrm{gm})\end{array}$ & $\underset{(g m)}{\mathrm{AOT}}$ \\
\hline \multicolumn{5}{|l|}{ Upper Layer: } \\
\hline 31.4 & 40.4 & 0.711 & 0.551 & 0.563 \\
\hline 59.4 & 40.8 & 0.714 & 0.867 & 0.556 \\
\hline 87.5 & 41.4 & 0.717 & 1.35 & 0.555 \\
\hline 115 & 42.2 & 0.724 & 2.11 & 0.557 \\
\hline \multicolumn{5}{|l|}{ Lower Layer: } \\
\hline 31.4 & 0.14 & 0.993 & 0.17 & 0.002 \\
\hline 59.4 & 0.53 & 1.01 & 0.49 & 0.008 \\
\hline 87,5 & 0.70 & 1.01 & 0.65 & 0.006 \\
\hline 115 & 0.55 & 1.01 & 0.52 & 0.004 \\
\hline
\end{tabular}

${ }^{a}$ Composition of the entire system, $40 \mathrm{ml}$ of $2 \% \mathrm{w} / \mathrm{w}$ AOT in $n$-octane plus added water.

${ }^{b}$ Specific gravity of pure $n$-octane $=0.7009$.

volume of the upper layer increases as the ratio of the moles of water per mole of $A O T$ of the entire system is increased, and parallel to this, the specific gravity also increases. By contrast, the volume of the lower layer increases to a maximum about midway through this region, and then decreases as the molar ratio of the entire system is increased, whereas the specific gravity remains virtually constant throughout. Note in Table III that changes in water content for both phases parallel these volume changes. Since about $0.63 \mathrm{ml}$ of water is required to give each increase in molar ratio and this amount cannot account for the increase in volume or water content of any one phase, it would appear that the added water partitions between the two phases up to the point where the separated phase begins to be resolubilized.

That a turbid phase appears and disappears upon the addition of water would seem to indicate that each increment of water results in a change in micellar organization, which in turn produces changes in water-solubilizing ability. Such changes in micellar size and shape for AOT in water-dodecane systems have been reported by Mathews and Hirschhorn (2) at relatively low molar ratios of water to AOT so that marked micellar changes in the present systems might be expected. Calculations based on data in Table 
TABLE IV

Upper Layer Specific Gravities of Water/ Aerosol OT/Hydrocarbon Solvent Sxstems AT $25^{\circ} \mathrm{C}$

\begin{tabular}{c|c|c}
\hline & $\frac{\text { Moles water }}{\text { mole AOT }}$ & $\begin{array}{c}\text { Specific } \\
\text { gravity }\end{array}$ \\
\hline $\begin{array}{c}\text { Water-AOT-n- } \\
\text { octane: } \\
\text { Second turbid re- } \\
\text { gion }\end{array}$ & 170 & \\
& 185 & 0.7025 \\
Water-AOT- & 0.7015 \\
dodecane: & Pure $n$-octane & 0.7009 \\
Turbid region & & \\
& 50 & 0.7509 \\
Water-AOT- & 65 & 0.7503 \\
hexadecane: & Pure dodecane & 0.7496 \\
Turbid region & & \\
& 20 & 0.7748 \\
& 30 & 0.7731 \\
& Pure hexadecane & 0.7723 \\
\hline
\end{tabular}

III reveal that the concentration of $\mathrm{AO}^{\prime} \mathrm{T}$ in the upper layer continues to decrease as water is added, whereas, concurrently, that in the lower layer first increases slightly and then decreases until the return of $A O T$ and water to the upper phase is complete. It is, most likely, these changes in AOT concentration which bring about the micellar changes responsible for the appearance and disappearance of turbidity; however, the system is actually much too complicated to draw any specific conclusions without further study. In the hydrocarbon phase, for example, an equilibrium can exist between AOT monomers, water dissolved in hydrocarbon, micellar AOT, micellar solubilized water, and hydrocarbon solvent; whereas in the separated aqueous phase this can involve $A O^{\prime} \mathrm{T}$ monomers, dissolved hydrocarbon, micellar AOT, and solubilized hydrocarbon. Since AOT is soluble in both water and hydrocarbon, an additional complicating factor may arise in that AOT may be dissolved in the micellar solubilized water and the micellar solubilized hydrocarbon.

Second Turbid Region in the Water/AOT/ Octane System and the Turbid Region in Water/AOT/Dodecane and Hexadecane Systems at $25^{\circ} \mathrm{C}$. The results of the determina- tion of the upper layer specific gravities at $25^{\circ} \mathrm{C}$ for all three systems (Table IV) indicate a trend for the specific gravity of the upper layer to approach more closely the value of the pure solvent as the molar ratio of the entire system is increased. Upon analysis of the upper and lower layers in the $n$-octane systems for AOT, it was found that there was no detectable amount in the upper layer, whereas all the AOT could be accounted for in the lower layer. Similar analysis of the upper layer in the dodecane system also indicated the absence of AOT in that layer. AOT analyses could not be performed on the lower layers of the dodecane and hexadecane systems because of their very small volumes nor on the upper layer of the hexadecane system because of the high boiling point $\left(324^{\circ} \mathrm{C}\right)$ of this solvent.

In comparing the amount of water solubilized at the first appearance of turbidity in all solvents (Table I), it was noted that with increasing solvent chain length, solubilization increased to a maximum in dodecane and then decreased. However, in view of the apparent differences in the mechanisms producing turbidity at this first transition point, and the similarity of the second turbidity point in $n$-octane and $n$-decane and the single turbidity point in the higher hydrocarbons, it might be better to compare solubilization tendencies at this latter transition point. As seen in Fig. 10, therefore, an increase in the solvent chain length produces a marked

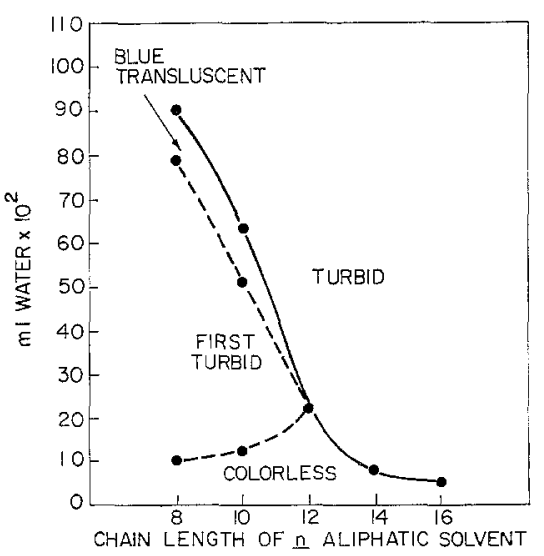

Fig. 10. Phase transitions in water $/ 2 \% \mathrm{w} / \mathrm{w}$ Aerosol OT $/ n$-aliphatic hydrocarbon solvent systems at $25^{\circ} \mathrm{C}$. 
reduction in the capacity for soluiblization of water. Further support for comparing the systems in this manner is the fact that an increase in temperature produces a tendency for the first region in the $n$-octane and $n$ decane systems to disappear (Figs. 4 and 7) leaving only common turbidity points. As seen in Fig. 8 (top), dodecane may be considered to be a transition solvent, in that plots of the data obtained at $35^{\circ} \mathrm{C}$ and $45^{\circ} \mathrm{C}$ show the same type of inverse temperature dependency as seen in the $n$-octane and $n$ decane systems, whereas the shape of the transition curve for dodecane at $25^{\circ} \mathrm{C}$ resembles that for the first phase transition seen with $n$-octane and $n$-decane at higher temperatures. Another similarity between the $35^{\circ} \mathrm{C}$ and $45^{\circ} \mathrm{C}$ transitions in dodecane and the transitions into the second turbid region in $n$-octane and $n$-decane is that the number of moles of water solubilized per mole of AOT increases with decreasing AOT concentrations in the more dilute solutions. At this point it is not clear exactly what is producing these significant solvent effects, but it is clear that even closely related hydrocarbon solvents cannot be grouped together merely as nonpolar solvents for such systems.

Effect of Salts on the Water/AOT/n-Octane System. As shown in Table V, salts dissolved in solubilized water produce reduction in solubilization at the transition from the colorless to the first turbid region, which is independent of salt and salt concentration up to $0.05 M$. However, transitions from the first turbid to the blue translucent region appear to be more dependent on salt concentration, as well as on the type of cation. It is particularly interesting to note that equivalent concentrations of sodium ion $\left(0.01 M \mathrm{NaCl}\right.$ and $\left.0.005 M \mathrm{Na}_{2} \mathrm{SO}_{4}\right)$ increase to the same extent the amount of water required to produce the blue translucent region, whereas $0.01 M \mathrm{LiCl}$ has a somewhat smaller effect. The greater influence of sodium ion is noticeable at $0.05 M$ concentration. Here resolubilization is completely blocked by sodium ion, whereas only at $1.0 \%$ AOT or higher is this effect seen with $0.05 \mathrm{M}$ $\mathrm{LiCl}$. The nonspecific effects of salts observed in the transition from the colorless to the first

\section{TABLE $\mathrm{V}$}

Effects of Salts and Aerosol OT Concentramion on the Amount of Water Required for Phase Transitions in a Water/Aerosol OT/n-OCTANE System at $25^{\circ} \mathrm{C}$

$\left(\mathrm{ml} \times 10^{2}\right)^{a}$

\begin{tabular}{|c|c|c|c|c|c|c|}
\hline$\underset{n-\mathrm{C} 8}{\mathrm{AOT}}$ & $\begin{array}{l}\text { Dist. } \\
\text { water }\end{array}$ & $\stackrel{0.01 M}{\mathrm{NaCl}}$ & $\underset{\mathrm{NaCl}}{0.05 \mathrm{M}}$ & $\underset{\mathrm{LiCl}}{0.01 M}$ & $\begin{array}{c}0.05 M \\
\mathrm{LiCl}\end{array}$ & $\begin{array}{l}0.005 M \\
\mathrm{Na}_{2} \mathrm{SO}_{4}\end{array}$ \\
\hline
\end{tabular}

Transition: Colorless to First Turbid Region

\begin{tabular}{l|r|r|r|r|r|r}
0.10 & 0.5 & 0.4 & 0.4 & 0.4 & 0.4 & 0.4 \\
0.25 & 1.3 & 1.1 & 1.1 & 1.1 & 1.1 & 1.1 \\
0.50 & 2.5 & 2.2 & 2.2 & 2.2 & 2.2 & 2.2 \\
1.0 & 5.0 & 4.7 & 4.7 & 4.7 & 4.7 & 4.7 \\
2.0 & 10.2 & 8.9 & 8.9 & 8.9 & 8.9 & 8.9
\end{tabular}

Transition: First Turbid to Blue Translucent Region

\begin{tabular}{l|r|r|l|r|r|r}
0.10 & 4.1 & 6.2 & $b$ & 5.7 & 7.9 & 6.2 \\
0.25 & 10.1 & 15.7 & $b$ & 14.2 & 19.5 & 15.2 \\
0.50 & 19.5 & 30.4 & $b$ & 28.2 & 37.9 & 30.9 \\
1.0 & 39.1 & 62.4 & $b$ & 55.0 & $b$ & 62.4 \\
2.0 & 78.9 & 125 & $b$ & 107 & $b$ & 117
\end{tabular}

Transition: Blue Translucent to Second Turbid Region

\begin{tabular}{l|r|r|r|r|r|r}
0.10 & 4.7 & 6.8 & $b$ & 6.3 & 8.2 & 7.0 \\
0.25 & 11.4 & 17.2 & $b$ & 15.7 & 20.4 & 16.7 \\
0.50 & 22.4 & 34.2 & $b$ & 30.2 & 39.9 & 32.4 \\
1.0 & 45.1 & 67.4 & $b$ & 62.4 & $b$ & 72.4 \\
2.0 & 90.3 & 135 & $b$ & 117 & $b$ & 127 \\
\hline
\end{tabular}

a Per $10 \mathrm{ml}$ AOT solution in $n$-octane, corrected for water solubility in $n$-octane at $25^{\circ} \mathrm{C}(19)$.

$b$ No transition observed.

turbid region may reflect a general competition of salt for water or a slight alteration in micelle structure; however, if the effect in these regions was on water activity alone one might expect lithium ion to have a greater effect because of its high hydration energy. In view of the greater effect of sodium ion, particularly at $0.05 \mathrm{M}$, some specific suppression of AOT dissociation may be occurring, and this could be a factor in determining micellar size and organization.

Water/AMA and Water/OOT Systems in $n$-Octane. Both OOT and AMA solutions in $n$-octane form turbid phases when a fraction of a mole of water per mole of surfactant is added. Increasing the temperature to $45^{\circ} \mathrm{C}$ has very little effect on this behavior. Further increments of water tend to produce the 
types of phase transitions seen with AOT in $n$-octane, particularly when the temperature is raised to $45^{\circ} \mathrm{C}$; however, the regions appear quite different and occur over a shorter range of molar ratios. For example, a relatively clear but viscous one-phase region in OOT occurs at $45^{\circ} \mathrm{C}$ at about 30 moles of water per mole of OOT, but only over a range of 3 moles of water per mole of OOT. At lower temperatures this region occurs, but it is never really clear. Solutions of AMA at $45^{\circ} \mathrm{C}$, on the other hand, produce a clear gel at a molar ratio of 25 . This gel extends over a range of 5 moles of water per mole of AMA and the becomes quite opaque. At $25^{\circ} \mathrm{C}$ and $35^{\circ} \mathrm{C}$ gels also appear but they are always quite opaque. In general, therefore, under comparable conditions AMA and OOT do not exhibit the ability to solubilize water to the same extent as AOT, and despite a limited tendency to show clearing of the system with increasing water content, they tend to produce rather viscous turbid systems.

It would seem that the major factor responsible for these differences is the rather bulky ethyl side chain associated with AOT and the straight alkyl chains of OOT and AMA. Both AMA and OOT being crystalline in the solid state because of strong chain interactions might be expected to exhibit similar behavior in the presence of water and produce large micellar aggregates which are not as soluble in $n$-octane. Indeed, even in the absence of water OOT and AMA exhibit very limited solubility in hydrocarbon solvents having more than eight carbons. On the other hand, just as the ethyl side chain apparently promotes the amorphous solid state character and liquid cyrstalline behavior of AOT when in contact with water it could produce the proper micellar structure which allows significant growth and reorganization of micelles in order to accommodate larger quantities of water and retain a fluid system.

More definitive quantitative estimates of hydrous micellar structure for the various surfactants are needed to explain the unique behavior of AOT in these systems; however, it is clear from these observations that the complex AOT/water/hydrocarbon systems are extremely interesting and worthy of further consideration as models of the more complex microemulsion systems,

\section{ACKNOWLEDGMENTS}

The authors wish to thank the Bristol-Myers Company for their generous support of this project.

\section{REFERENCES}

1. Peri, J. B., J. Am. Oil Chemists Soc. 35, 110 (1958).

2. Mathews, M. B., and Hirschiorn, E., $J$. Colloid Sci. 8, 86 (1953).

3. Kitahara, A., Kobayashi, T., and TachiBana, T., J. Phys. Chem., 66, 363 (1962).

4. Philipoff, W., J. Colloid Sci. 5, 169 (1950).

5. Aebi, C. M., and Wiebush, J. R., J. Colloid Sci. 14, 161 (1959).

6. Kitahara, A., Ishikawa, T., and Tanimori, S., J. Colloid and Interface Sci. 23, 243 (1967).

7. Kitahara, A., and Kon-No, K., J. Phys. Chem. 70, 3394 (1966).

8. Higuchi, W. I., AND Misra, J., J. Pharm. Sci. $51,455(1962)$.

9. Cooke, C. E., ANd Schulman, J. H., In Ekwall, P., Groth, K., and Runnstrom-Reio, V. eds., "Surface Chemistry," p. 231. Academic Press, New York, 1965.

10. Balmbra, R. R., Clunne, J. S., and Goodman, J. R., Proc. Roy. Soc. (London) A285, 534 (1965).

11. Gilchrist, C. A., Rogers, J., Steen, G., VAAL, E, G., AND Winsor, P. A., J. Colloid and Interface Sci. 25, 409 (1967).

12. Corkill, J. M., Goodman, J. F., and Walker, T., Trans. Faraday Soc. 61, 589 (1965).

13. Robson, P., Procter and Gamble, Ltd. (England), Personal communication, 1965.

14. Wilitiams, E. F., WoodberRy, N. T., AND Dixon, J. K., J. Colloid Sci. 12, 452 (1957).

15. Shinoda, K., "Colloidal Surfactants," p. 81. Academic Press, New York, 1963.

16. Gillap, W. R., Weiner, N. D., and Gibaldi, M., J. Am. Oil Chemists Soc. 44, 71 (1967).

17. Aveyard, R., and Haydon, D. A., Trans. Faraday Soc. 61, 2255 (1965).

18. "Handbook of Chemistry and Physics," 42nd ed. Chemical Rubber Publishing Co., Cleveland, Ohio, 1961.

19. Englin, B. A., Plate, A. F., Tugolukov, V. M., and Pryanishnikova, M. A., Khim. $i$ Teknol. Topliv $i$ Masel 10 (9), 42 (1965), Russ., Chem. Abstr. 63, 14608f (1965). 